\title{
Análisis de requerimiento de mayor ancho de banda del enlace de internet para la estación de radio institucional para cubrir la demanda de usuarios
}

\author{
Requirement analysis of higher internet bandwidth for institutional radio \\ station to fulfill users demand
}

León Felipe Austria González UNIVERSIDAD TECNOLÓGICA DE LA SIERRA HIDALGUENSE leon_austria@hotmail.com

Raymundo Sergio Noriega Loredo UNIVERSIDAD TECNOLÓGICA DE LA SIERRA HIDALGUENSE

snoriegal@gmail.com

\section{Resumen}

La Internet ofrece un futuro promisorio a los comunicadores sociales y productores radiofónicos que buscan un camino alternativo para hacer radio. Les da a los ingenieros en TIC la posibilidad de desarrollar nuevas tecnologías para la transmisión de audio por la web (streaming), diversas oportunidades en varias áreas del conocimiento, nuevas posibilidades comunicativas, mayor cobertura geográfica, nuevos formatos radiales, interactividad, ampliación de audiencias y facilidad de montaje pues no se requiere licitación de espectro y el equipo es de menor costo.

La presente es una investigación sobre el análisis del ancho de banda del enlace de Internet asignado a la estación de radio para poder ofrecer un servicio de calidad. En dicho trabajo se obtuvieron los promedios de las mediciones de bajada y subida de datos dentro de la jornada de trabajo diaria de lunes a viernes (12 muestras por día), las cuales nos arrojan un promedio de ancho de banda real actual para la transmisión de la barra programática de $725 \mathrm{Kbps}$. Con este ancho de banda solo se puede atender a 22 usuarios simultáneamente 
con una velocidad de transmisión de $32 \mathrm{Kbps}$ a un canal (mínima de transmisión), la cual ya no es suficiente para atender la demanda actual. Los usuarios potenciales para conectar a diario simultáneamente son 31 alrededor del mundo comprendidos por 18 países. Para atender la demanda actual y ofrecer un servicio de calidad de streaming (transmisión de programas radiofónicos) se requiere incrementar y contar con un ancho de banda de 4Mbps (2 Mbps de subida y 2 Mbps de bajada de datos si fuera simétrica).

Palabras clave: Ancho de Banda, streaming, transmisión, bajada y subida de datos.

\section{Abstract}

Internet offers a promising future to social communicators and radio producers who seek an alternative pathway to make radio. It gives Information and Communications Technology (ICT) engineers the possibility of developing new technologies for audio transmission through the web (streaming), diverse opportunities in several areas of knowledge, new communicative possibilities, greater geographic coverage, new radio formats, interactivity, larger audiences and ease setup as a bid is not required for spectrum and equipment is lower cost.

This is a research on the analysis of the Network Bandwidth assigned to a radio station to offer a quality service. In such work the average measurements were calculated for of descent and ascent of data within the daily working day from Monday to Friday (12 samples per day), which shed us an average of current actual bandwidth for the programmes transmission of $725 \mathrm{Kbps}$. With this bandwidth only 22 users can attend simultaneously with a transmission rate of $32 \mathrm{Kbps}$ to one channel (minimum of transmission), which is already not enough to meet the current demand. The potential users to connect daily and simultaneously are 31 around the globe spread over 18 countries. To meet the current demand and offer a quality streaming service (transmission of radio programmes), an increase and access to a bandwidth of $4 \mathrm{Mbps}$ is required (2 $\mathrm{Mbps}$ of upload and 2 Mbps of data down if it were symmetrical.

Key Words: Bandwidth, streaming, descent and ascent of data transmission. 
Fecha recepción: Marzo 2014 Fecha aceptación: Junio 2014

\section{Introducción}

Con la llegada de nuevas tecnologías de la información y la comunicación, la posibilidad de expansión ha crecido tanto para los comunicadores y productores de radio como para los desarrolladores de software e ingenieros en TIC. La Internet despeja un presente y abre un futuro promisorio.

La radio en frecuencia modulada $(\mathrm{FM})$ y amplitud modulada $(\mathrm{AM})$ ya no tiene cabida para concesionar más frecuencias porque el espectro electromagnético está saturado y monopolizado. Por ese motivo, los comunicadores sociales y productores radiofónicos deben buscar un camino alternativo para hacer radio, donde los ingenieros en TIC tengan la posibilidad de desarrollar nuevas tecnologías para la transmisión de audio por la web (streaming).

\section{Justificación}

Desde el punto de vista académico, la radio por Internet se vuelve otro recurso académico para estimular el desarrollo de las academias de las instituciones, el desarrollo profesional y el sector productivo, contribuyendo así al progreso del país. Por ser este un canal interactivo, altamente flexible y autónomo, también contribuye a la descentralización del conocimiento, permitiendo al usuario utilizar su creatividad al generar su propio contenido y expresarlo libremente.

Asimismo, la presente investigación sobre el análisis del requerimiento de mayor ancho de banda para el enlace de Internet a la estación de radio, tiene la finalidad de atender un proyecto real, aprobado por la sociedad y comunidad regional, nacional y del extranjero (por ejemplo, los paisanos que por algún motivo emigraron a otros países y utilizan la radio para acercarse a sus familiares y amigos). Con la creación e implementación de la estación de radio en Internet "Radio UT de la Sierra Hidalguense", se contribuye al desarrollo 
indirecto de los estudiantes de las carreras de Tecnologías de la Información y Comunicación, de músicos, aficionados a la radio y a la misma Internet con contenido de calidad. De esa manera, la Institución se vincula con el sector educativo, el productivo y la población regional de Sierra Alta del estado de Hidalgo, posicionando a la UTSH a la vanguardia en las telecomunicaciones.

\section{Desarrollo}

Para obtener los promedios de las mediciones de bajada y subida de datos se hicieron 12 mediciones diarias desde las 8 am a las 7 pm. Se tomó este horario como base ya que es cuando se registra mayor cantidad de usuarios conectados a Internet y hay personal en la radio para su monitoreo.

Durante el periodo de una semana se obtuvieron las siguientes mediciones (ver Tabla 1).

Tabla 1. Promedio de las mediciones de ancho de banda en Kbps de lunes a viernes.

\begin{tabular}{|c|c|c|}
\hline Hora & Bajada & Subida \\
\hline $8 \mathrm{am}$ & $815 \mathrm{Kbps}$ & $1355 \mathrm{Kbps}$ \\
\hline $9 \mathrm{am}$ & $260 \mathrm{Kbps}$ & $540 \mathrm{Kbps}$ \\
\hline $10 \mathrm{am}$ & $920 \mathrm{Kbps}$ & $660 \mathrm{Kbps}$ \\
\hline $11 \mathrm{am}$ & $680 \mathrm{Kbps}$ & $570 \mathrm{Kbps}$ \\
\hline $12 \mathrm{am}$ & $860 \mathrm{Kbps}$ & $785 \mathrm{Kbps}$ \\
\hline $1 \mathrm{pm}$ & $445 \mathrm{Kbps}$ & $650 \mathrm{Kbps}$ \\
\hline $2 \mathrm{pm}$ & $753 \mathrm{Kbps}$ & $670 \mathrm{Kbps}$ \\
\hline $3 \mathrm{pm}$ & $520 \mathrm{Kbps}$ & $875 \mathrm{Kbps}$ \\
\hline $4 \mathrm{pm}$ & $465 \mathrm{Kbps}$ & $660 \mathrm{Kbps}$ \\
\hline $5 \mathrm{pm}$ & $290 \mathrm{Kbps}$ & $500 \mathrm{Kbps}$ \\
\hline $6 \mathrm{pm}$ & $650 \mathrm{Kbps}$ & $740 \mathrm{Kbps}$ \\
\hline $7 \mathrm{pm}$ & $340 \mathrm{Kbps}$ & $415 \mathrm{Kbps}$ \\
\hline
\end{tabular}

Para la medición del máximo número de usuarios se utilizó el siguiente razonamiento: 
$\mathbf{M}$ = Máximo de usuarios que pudieran conectarse simultáneamente al servidor a una hora determinada (conexiones de streaming).

$\mathbf{A}=$ Medición del ancho de banda disponible a esa hora (Kbps).

$\mathbf{T}=$ Trafico de otros servicios de red a la internet a esa hora (Kbps).

$\mathbf{S}=$ Velocidad del servicio ofrecido de streaming (32 Kbps).

D = Número de días muestreados (Lunes - Viernes).

D

\section{$M=\left[\Sigma\left(A_{i}-T_{i}\right) / S\right] / D$}

\section{$\mathrm{i}=1$}

En la Figura 1 podemos ver la grafica del comportamiento del ancho de banda que se tiene asignada a la estación de radio Institucional. Como se observa en las mañanas en el horario de 8 a 9 am, se dispone de un ancho de banda casi libre como es de suponer por los pocos usuarios conectados a la Internet en la universidad (persona y alumnos); entre las 10 y 12 am hay mayor demanda de usuarios y no es posible ofrecer el servicio a más de 22 personas, además de que el servicio ofrecido no es de buena calidad (32 Kbps). A lo largo del día el ancho de banda ofrecido está en promedio en $725 \mathrm{Kbps}$, ya que es cuando los usuarios de la red e Internet de la universidad están conectados y compartiendo nuestro enlace, y por las tardes se ofrece menor ancho de banda en las transmisiones de programas, lo que genera quejas por parte de radioescuchas y locutores de la estación. En suma, por las tardes es cuando mayor número de personas en la Institución se conecta al Internet, además de que el servicio ofrecido por la compañía que lo suministra (TELMEX) no está garantizando el ancho de banda ofrecido. 


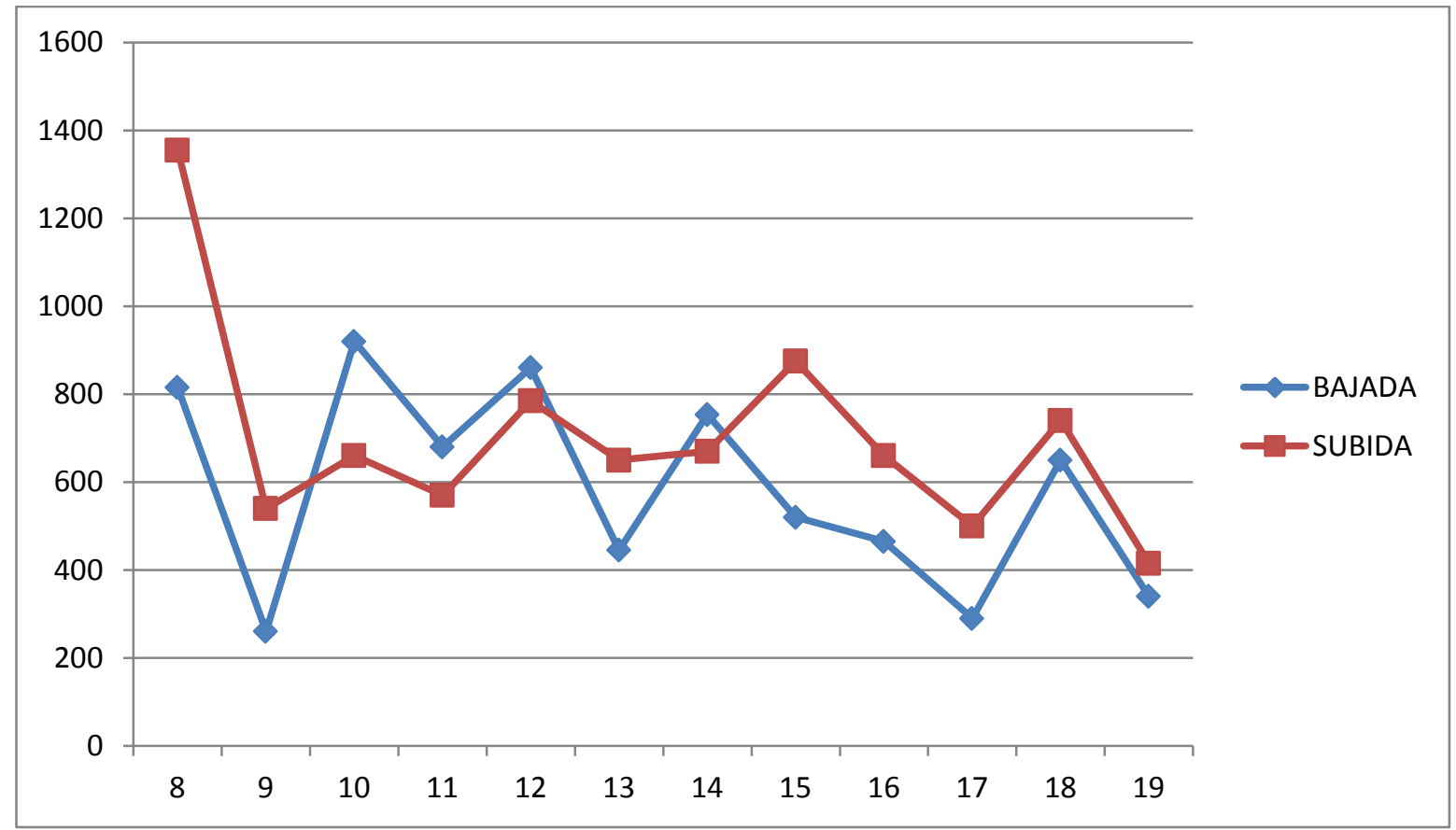

Figura 1. Análisis de ancho de banda (Kbps) del enlace de Internet de la radio institucional.

El máximo número de posibles usuarios conectados en forma simultánea se muestra en la tabla 2 .

Tabla 1. Promedio de usuarios máximos a conectarse en diferentes horarios de lunes a viernes.

\begin{tabular}{|c|c|}
\hline Hora & $\begin{array}{c}\text { Máximo de } \\
\text { usuarios }\end{array}$ \\
\hline $8 \mathrm{am}$ & $\mathbf{4 2}$ \\
\hline $9 \mathrm{am}$ & $\mathbf{1 7}$ \\
\hline $10 \mathrm{am}$ & $\mathbf{2 1}$ \\
\hline $11 \mathrm{am}$ & $\mathbf{1 8}$ \\
\hline $12 \mathrm{am}$ & $\mathbf{2 5}$ \\
\hline $1 \mathrm{pm}$ & $\mathbf{2 0}$ \\
\hline $2 \mathrm{pm}$ & $\mathbf{2 1}$ \\
\hline $3 \mathrm{pm}$ & $\mathbf{2 7}$ \\
\hline $4 \mathrm{pm}$ & $\mathbf{2 1}$ \\
\hline $5 \mathrm{pm}$ & $\mathbf{1 6}$ \\
\hline $6 \mathrm{pm}$ & $\mathbf{2 3}$ \\
\hline $7 \mathrm{pm}$ & $\mathbf{1 3}$ \\
\hline
\end{tabular}

Las mediciones anteriores arrojan un promedio de ancho de banda real actual para la transmisión de la barra programática de $725 \mathrm{Kbps}$. Con este ancho de banda solo se pueden 
atender en promedio a 22 usuarios simultáneamente con una velocidad de transmisión de 32Kbps a un canal (mínima de transmisión), la cual es suficiente para atender la demanda actual.

Los usuarios potenciales para conectarse diariamente de manera simultánea son 31 alrededor del mundo (Ver Figura 2), comprendidos en 18 países (Ver Tabla 3). Para atender la demanda actual y ofrecer un servicio de calidad de streaming (transmisión de programas radiofónicos) se requiere incrementar y contar con un ancho de banda de 4Mbps (2 Mbps de subida y 2 Mbps de bajada de datos si fuera simétrica).

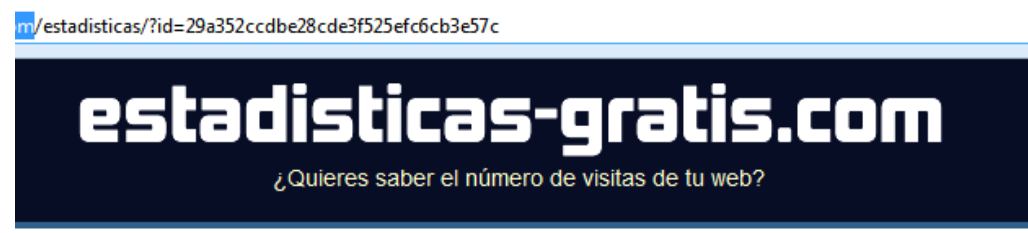

\section{Estadísticas Gratis de http://radio.utsh.edu.mx}

\begin{tabular}{ll}
\multicolumn{2}{l}{ Resumen de las visitas } \\
Páginas vistas totales & Visitas de la semana actual \\
7.864 & 101 \\
Páginas vistas hoy & Visitas de la semana anterior \\
0 & 104 \\
Páginas vistas ayer & Media de la semana actual \\
31 & 101
\end{tabular}

Figura 2. Imagen de la página de estadísticas para el dominio de la radio.

Tabla 3. Estadística en porcentajes de los usuarios de los países que nos escuchan (estadisticas-gratis.com). 


\begin{tabular}{|c|c|c|}
\hline \multicolumn{3}{|c|}{ TOP 20 de países } \\
\hline \#1. 四 México & 6.376 & $81,31 \%$ \\
\hline \#2. Estados Unidos & 1.280 & $16,32 \%$ \\
\hline \multirow[t]{2}{*}{ \#3. 四 Perú } & 58 & $0,74 \%$ \\
\hline & 26 & $0,33 \%$ \\
\hline \#5. 四 Canadá & 22 & $0,28 \%$ \\
\hline \#6. 回 Rusia & 21 & $0,27 \%$ \\
\hline \#7. 回 Uruguay & 20 & $0,26 \%$ \\
\hline \# 8. $\square$ España & 5 & $0,06 \%$ \\
\hline \#9. Argentina & 4 & $0,05 \%$ \\
\hline \#10. $⿴$ Colombia & 3 & $0,04 \%$ \\
\hline \#11. Bolivia & 3 & $0,04 \%$ \\
\hline \# 12. 포 República Dominicana & 3 & $0,04 \%$ \\
\hline \#13. Alemania & 3 & $0,04 \%$ \\
\hline \#14. India & 3 & $0,04 \%$ \\
\hline \#15. 0 Venezuela & 2 & $0,03 \%$ \\
\hline \# 16. Unión Europea & 1 & $0,01 \%$ \\
\hline \#17. Indonesia & 1 & $0,01 \%$ \\
\hline \#18. 믄 Chile & 1 & $0,01 \%$ \\
\hline
\end{tabular}

\section{Conclusión}

Para poder garantizar la calidad del servicio radiofónico por Internet que ofrece la UTSH con respecto a la cantidad de usuarios que se conectan y siguen este proceso de comunicación, es necesario que se amplíe la cobertura con un ancho de banda de 4Mbps. Con dicha cobertura se busca aprovechar el ancho de banda y mejorar los servicios que ofrecen los programas radiofónicos de divulgación de la salud, la ciencia y la tecnología, la educación, el impacto social, la cultura, las artes, las tradiciones y el rescate de la lengua indígena.

\section{Bibliografía}

http://www.estadisticas-gratis.com/estadisticas/?id=29a352ccdbe28cde3f525efc6cb3e57c http://www.utsh.edu.mx/php/inicio.php 